\title{
Intractable Facial Pain and Numb Chin due to Metastatic Esophageal Adenocarcinoma
}

\author{
Foad Elahi $^{\mathrm{a}}$ Whitney Luke ${ }^{\mathrm{a}}$ Fazel Elahi $^{\mathrm{b}}$ \\ ${ }^{a}$ Center of Pain Medicine, University of Iowa, Iowa City, Iowa, USA; ${ }^{\mathrm{b}}$ Cancer Center, Tehran \\ University, Tehran, Iran
}

\section{Key Words}

Facial pain · Numb chin syndrome $\cdot$ Inferior alveolar nerve · Esophageal cancer ·

Adenocarcinoma

\begin{abstract}
The etiologies of facial pain are innumerable, thus facial pain misdiagnosis and resultant mismanagement is common. Numb chin syndrome presents with hypoesthesia and/or anesthesia in the dermatomal distribution of the inferior alveolar or the mental nerve. In this case report, we will discuss a case of intractable facial pain in a 57-year-old male with a history of esophageal adenocarcinoma who was initially misdiagnosed and treated as trigeminal neuralgia. During clinical examination, the loss of sensation in the inferior alveolar nerve distribution was identified and led to the diagnosis of mandibular metastasis. The details of the clinical presentation will be discussed in the context of accurate identification and diagnosis. Focal radiation to the metastatic location along with sphenopalatine ganglion radiofrequency ablation and medication management provided significant pain relief. This case report provides additional information to the current medical knowledge and it enhances the clinical vigilance of the clinicians when they encounter similar cases. We concluded that patients with a history of neoplasms who present with atypical symptoms of facial pain should undergo further investigation with advanced imaging. Targeted treatment based on an accurate diagnosis is the foundation of pain management. $02014 \mathrm{~S}$. Karger AG, Basel
\end{abstract}

\section{Introduction}

Facial pain may be due to many etiologies, including neurological, vascular, or dental in origin. Patients will often make the first attempt at diagnosis in that he or she chooses to 
Elahi et al.: Intractable Facial Pain and Numb Chin due to Metastatic Esophageal Adenocarcinoma

consult either a dentist or the primary doctor. Neurological and vascular causes of facial pain are rare compared to dental and temporomandibular joint pathologies.

Facial pains with the following clinical diagnostic labels are easily recognizable by most clinicians: idiopathic trigeminal neuralgia (with normal neurological examination), trigeminal neuropathic pain resulting from injury to the trigeminal nerve and trigeminal deafferentation pain (i.e. peripheral nerve ablation, gangliolysis, or rhizotomy), postherpetic neuralgia, or trigeminal neuralgia resulting from multiple sclerosis [1].

Interchangeably, numbness of the chin in the literature is referred to as numb chin syndrome. Oftentimes, it can be the first sign of metastases in a patient with previously diagnosed cancer.

The inferior alveolar nerve is the terminal sensory division of the mandibular branch of the trigeminal nerve. Numbness of the lip and chin, the lower teeth and gum can be due to the inferior alveolar nerve or mental nerve neuropathy.

Malignant processes cause numb chin syndrome and have most often been attributed to metastatic breast cancer, thyroid cancer and lymphoma. Primary breast and thyroid cancer constitute the majority of these metastases at $20-30 \%$ and $15 \%$, respectively. Other malignancies, which are most commonly associated with numb chin syndrome, include lung cancer, prostate adenocarcinoma, multiple myeloma, and lymphoproliferative processes such as lymphoma. Numb chin syndrome may either be due to a compression of nerve tissue secondary to the tumor, such as at the trigeminal nerve root, or perineural spread of metastatic disease [2].

In this article, we describe a rare case with a few important and unique aspects. Intractable facial pain was the patient's main complaint for many months. The neurological deficit on the inferior alveolar nerve distribution was discovered relatively late in the course of the illness. Numbness of the chin was the main guidance for proper investigation and treatment. To the best of our knowledge, the adenocarcinoma metastases to the mandibular bone and involvement of the inferior alveolar nerve have not been previously reported in the literature.

\section{Case Report}

A 57-year-old male with stage IV metastatic esophageal adenocarcinoma who had completed a full course of chemotherapy and localized esophageal radiation about 6 months before, presented with complaints of left-sided facial pain for 3 months. The patient's pain had been progressively worsening and was significant during his chewing of solid foods, resulting in weight loss interpreted as a result of poor oral intake. The pain was described as sharp, lancinating, and throbbing, radiating to the left temple and cheek. The patient rated the severity of the facial pain as 8 out of 10 on the Numerical Pain Rating Scale.

The workup prior to his visit included a brain MRI and a maxillofacial CT that focused on the trigeminal nerve distribution in the brain, and facial sinuses identified no neural element involvement, metastases or bony lesions. A PET scan of the entire body at an outside hospital showed a hypermetabolic mass in the distal esophagus consistent with known adenocarcinoma.

With the prior diagnosis of trigeminal neuralgia, the patient had been on methadone, hydrocodone, and carbamazepine, prescribed by other providers. This combination of medications had failed to improve the pain severity. He was then referred to the center of pain management with the diagnosis of trigeminal neuralgia and continuing intractable facial pain. 
Elahi et al.: Intractable Facial Pain and Numb Chin due to Metastatic Esophageal Adenocarcinoma

On physical examination, the patient was in considerable distress due to the facial pain. He had a palpable trigger point over the left masseter muscle. The temporomandibular joint range of motion was within normal limits and with no associated pain. A sensory examination revealed a decreased sensation to light touch and pinprick over the left chin, the lower lip, and the lower oral mucosa, consistent with the distribution of the left inferior alveolar nerve. There was no facial asymmetry, and no other cranial nerve deficits were observed. We decided to perform a localized mandibular CT scan, on which we localized a metastatic lesion in the mandibular bone with the involvement of the inferior alveolar nerve (fig. 1).

Based on this new imaging, we reviewed his prior maxillofacial CT and PET scans. We paid specific attention to the left mandibular region because the outside facility had not mentioned an increased uptake in that region. Upon secondary review, focal uptake without any evidence of a bony lytic lesion in the left mandibular angle was noted, which likely represented evidence of early metastasis that was overlooked by prior image reading (fig. 2).

A referral to radiation oncology was issued for the localized radiation therapy of the left mandibular lesion. As part of the palliation, we decided to perform a left sphenopalatine ganglion block, which dramatically decreased the pain. The patient then received radiofrequency ablation of the left sphenopalatine ganglion based on his responses to the local anesthetic block. The combination of medications, radiofrequency, and subsequent localized radiation to the lesion provided significant pain relief. During his follow-up, he reported a pain reduction to $80 \%$ and was able to tolerate chewing the permitted semi-solid foods without pain.

\section{Discussion}

As stated in the Introduction, facial pain misdiagnosis and resultant mismanagement is common. It has been shown that a combination of CT imaging of the brain, skull base, and mandible in conjunction with CSF analysis yielded a diagnosis in $89 \%$ of patients with numb chin syndrome and known malignancy. However, in a patient who has a constant increasing severity of pain or unchanged symptoms such as swelling, neurologic signs, and/or marked trismus, it has been recommended to reassess and reimage if the symptoms do not respond to a trial of conservative therapy [3]. Once a diagnosis is obtained, localized radiation therapy could potentially help alleviate the symptoms.

The outside imaging, including the maxillofacial CT and PET scans, was reviewed by another radiologist. He paid specific attention to the left mandibular region as no mention had been made at the outside facility regarding the increased uptake in that region. Upon secondary review, focal uptake without evidence of a bony lytic lesion in the left mandibular angle was noted in the PET scan, which likely represented evidence of early metastasis.

Upon review of the literature, there is 1 documented case of numb chin syndrome secondary to esophageal squamous cell carcinoma [3]. However, there are no documented cases of esophageal adenocarcinoma with metastases to the mandible as in our case.

\section{Conclusion}

Neurological deficits in the head and neck area in a patient with a known malignancy should be considered as metastatic in origin until thorough workup has been completed. The evaluation should include CT or MRI with a specific focus based on the clinical findings and PET scan. 
For a patient with well-known malignancy who presents with new facial pain, if initial imaging is negative, it is strongly recommended to reassess and reimage if the symptoms do not respond to a trial of conservative therapy over a certain time period.

This case report provides additional information to the current medical knowledge as it enhances the clinical vigilance of the clinicians when they encounter similar cases. In conclusion, patients with a history of neoplasms, who present with atypical symptoms of facial pain, should undergo further investigation with advanced imaging. Targeted treatment based on an accurate diagnosis is the foundation of appropriate pain management.

\section{References}

1 Elahi F, Ho KW: Anesthesia dolorosa of trigeminal nerve, a rare complication of acoustic neuroma surgery. Case Rep Neurol Med 2014;2014:496794.

-2 Lossos A, Siegal T: Numb lip syndrome in cancer patients: etiology, response to treatment, and prognostic significance. Neurology 1992;42:1181-1184.

3 Huntley TA, Wiesenfeld D: Delayed diagnosis of the cause of facial pain in patients with neoplastic disease: a report of eight cases. J Oral Maxillofac Surg 1994;52:81-85.

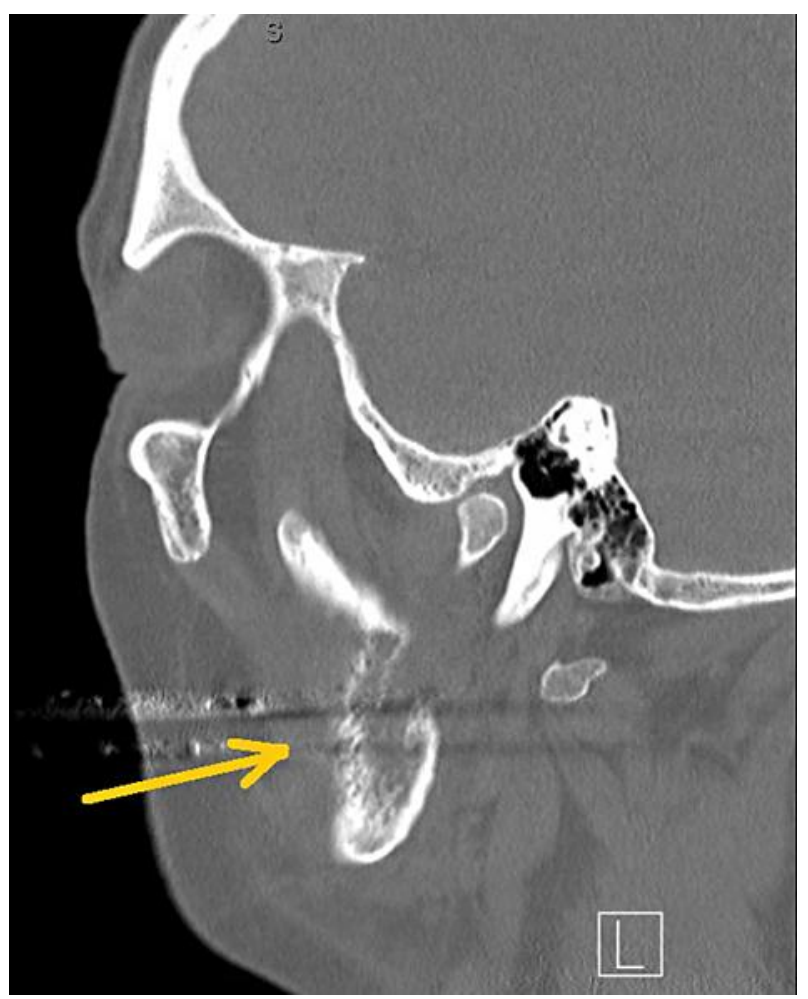

Fig. 1. Mandibular CT scan: the arrow shows the localized lytic lesion. 


\section{Case Reports in Oncology}

Case Rep Oncol 2014;7:828-832

DOI: $10.1159 / 000369785$

lahi et al.: Intractable Facial Pain and Numb Chin due to Metastatic Esophagea

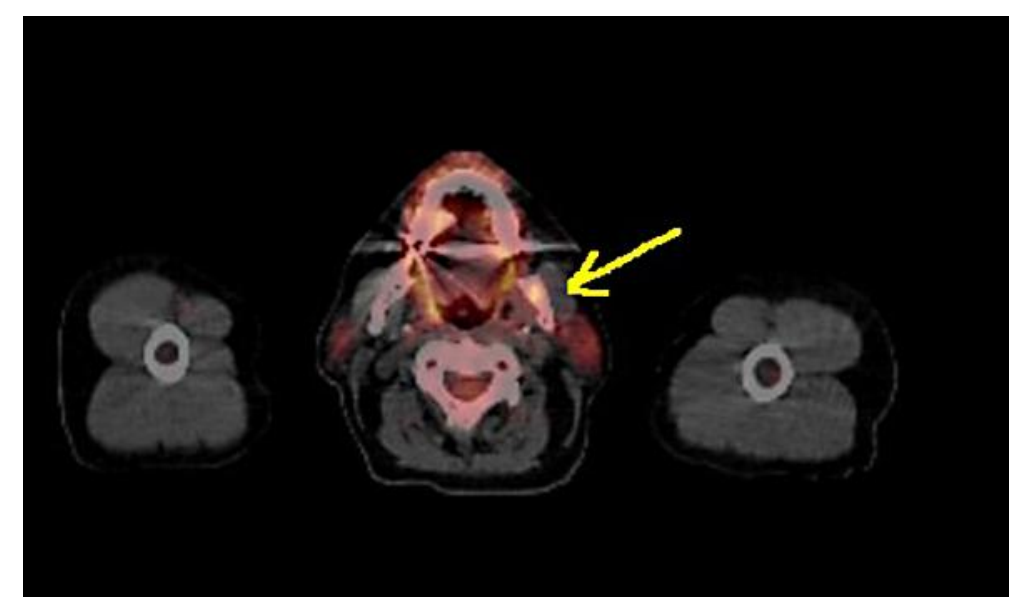

Fig. 2. PET imaging: the arrow shows the tiny focus of uptake in the left mandibular angle. 